Revue d'histoire de l'enfance « irrégulière »

Le Temps de l'histoire

2| 1999

Cent ans de répressions des violences à enfants

\title{
La lacune et le remède : la tâche du législateur républicain
}

Annie Stora-Lamarre

\section{Q OpenEdition \\ 12 Journals}

Édition électronique

URL : http://journals.openedition.org/rhei/32

DOI : $10.4000 /$ rhei.32

ISBN : 978-2-7535-1639-7

ISSN : $1777-540 \mathrm{X}$

Éditeur

Presses universitaires de Rennes

Édition imprimée

Date de publication : 15 novembre 1999

Pagination : 47-58

ISSN : 1287-2431

Référence électronique

Annie Stora-Lamarre, "La lacune et le remède : la tâche du législateur républicain », Revue d'histoire de l'enfance « irrégulière » [En ligne], 2 | 1999, mis en ligne le 30 juillet 2010, consulté le 04 décembre 2020. URL : http://journals.openedition.org/rhei/32 ; DOI : https://doi.org/10.4000/rhei.32 


\section{La lacune et le remède : la tâche du législateur républicain}

Ruiner les fondements du code pénal de 1810, code à la fixité sans espoir, s'ouvrir au droit international au moment où l'on déplore le retard de la France en regard de l'avance des codes hongrois et hollandais qui proportionnent la peine à l'âge de la victime et à la gravité du mal corporel, pénétrer au domicile privé du criminel où la victime ne peut pas se défendre, où sa plainte ne se fait pas entendre, permettre aux associations charitables d'être investies du droit de citer directement en police correctionnelle les auteurs de violences envers les enfants, telles sont les lacunes pointées par la loi du 19-21 avril 1898 sur la répression des violences, voies de faits, actes et cruauté et attentats commis envers les enfants. La lacune, c'est l'espace vide dans lequel s'engouffre tout un discours de déploration sur des affaires tragiques : Villain (26 février 1892), Fiévet (20 avril 1892), Machicoine (12 août 1892), Lefebvre (29 septembre 1892). La République refuse la fatalité du mal au nom de la morale et du droit. Les juristes de la fin du siècle guettent dans la jurisprudence la fissure par où tromper la longue attente imposée par le Parlement. Les grandes théories jurisprudentielles ne doivent pas être de simples survivances du passé, mais marquer une anticipation sur l'avenir et constituer ainsi une création intégrale. ${ }^{(2)}$ Au Parlement, le législateur cherche un jalonnement de route en renforçant les pièces de l'armature législative des lois de protection de l'enfance. Quel récit la loi du 19 avril 1898 inscrit-il entre la lacune et le remède?

\section{Annie Stora- Lamarre $^{(1)}$}

(1) Maître de conférences, Université de Besançon.

(2) E. ESMEIN,

"La jurisprudence et la doctrine”, Revue trimestrielle de droit civil, tome 1 , 1903, p. 5. 
(3) Jean-François LAE, L'Instance de la plainte. Une histoire politique et juridique de la souffrance, Paris, Descartes et Cie, 1996.

(4) Raymond

SALEILLES, Les Accidents du travail et la responsabilité civile, 1897, p. 4.

(5) François EWALD,

L'Etat providence, Paris, Grasset, 1986, p. 311.

(6) L'article 309 est inapplicable lorsque seules les marques et non la maladie produite ont duré plus de vingt jours. Cass. crim., 17 décembre 1819.

\section{DANS LES BRISURES DES CODES}

La loi de répression de violences à enfants apparait à un moment où le droit social naissant tente de réparer le gâchis de l'industrialisation. Faire autre chose du vivant que de le détruire, c'est la puissante protestation de la fin du XIXe siècle qui produira l'instance de la plainte, cet appel à disposer entièrement de soi-même contre tous les assauts inquisiteurs, punitifs ou sacrificiels. ${ }^{(3)}$ La pensée juridique de la fin du XIXe siècle prend en compte la souffrance du corps blessé. La loi du 9 avril 1898, concernant la responsabilité des accidents dont les ouvriers sont victimes dans leur travail, déplace l'idée de faute à celle de réparation. ${ }^{(4)}$ Le parlement avait institué, en dehors du code civil, un droit nouveau des obligations : un premier code de l'accident. L'accident, sorte de point aveugle du code civil, se trouvait juridiquement objectivé comme tel. La loi veut briser le terrible engrenage de la violence causée par la rançon du machinisme et de l'industrie moderne, où l'ouvrier n'est qu'un instrument passif au milieu d'autres moteurs purement mécaniques. Elle conférait un droit, indépendamment d'une analyse de ces causes. ${ }^{(5)}$ La même logique anime la loi du 19 avril 1898. Julien Goujon (1854-1912), qui en est un des artisans, se préoccupe de la législation industrielle et ouvrière en proposant une réduction des heures de travail dans les manufactures. Il dénonce aussi les carences du code pénal (articles 309, 310, 311 anciens) ${ }^{(6)}$ et exige que la matérialité des faits susceptibles d'entraîner des risques pouvant conduire à la mort entre dans le domaine de l'activité législative.

C'est, en effet, dans la perte des ancrages de ce qui dit le juste, le définitif que s'édifie la loi de 1898. Les juristes ont fabriqué le processus de naturalisation de la réforme, au sens de la prise en compte des changements nécessaires dans l'espace social de la fin du siècle. Le sort des déchus, bâtards, filles séduites, criminels, pères incapables, enfants martyrs ou coupables apparait impossible dans l'inertie du droit ou la formulation d'abstraites spéculations. Les codes du début du XIXe siècle n'apparaissent que comme le pâle reflet d'une symbolique qui expire. Lorsque la société est barbare, elle est soumise à la religion de la lettre; le droit est emprisonné dans le matérialisme de la forme, le symbole règne 
et domine. Les époques civilisées ont pour mission de débarrasser le droit de ces entraves, de l'élever au dessus des mots, de placer l'idée au-dessus de la forme. Il faut émanciper le droit, renverser toutes les formes dont le symbole est la manifestation expressive, arracher le code à la rigidité des textes, infuser les règles de la solidarité et de l'équité lorsque la vie collective fait entendre ses protestations. La loi nouvelle de la famille ne devra plus être la loi de l'homme, c'est-à-dire celle du mari et celle du père, mais devra être la loi de tous les individus composant le groupe familial. Ces temps nouveaux annoncent la fin des dogmes; la loi n'est pas immuable.

À la fin du siècle, plus précisément vers les années 1880, l'Ecole de l'exégèse achève son œuvre. Le premier trait distinctif, le trait fondamental de l'Ecole de l'exégèse, c'est le culte du texte de loi. ${ }^{(7)}$ Les juristes, spécialement les civilistes, se cantonnent rigoureusement dans le domaine d'action constitué par le code civil : "Les textes avant tout! ». Or, les lois sont toujours à l'état de remaniement, de transformation; elles sont des "réverbératrices". ${ }^{(8)}$ Pour le juriste allemand Ihering, toutes les grandes conquêtes qu'enregistre l'histoire du droit - abolition de l'esclavage et de la servitude personnelle, droit à la propriété foncière et industrielle, liberté des croyances - ont dû être remportées au prix de luttes ardentes, souvent continuées pendant des siècles. "Le droit, c’est bien Saturne dévorant ses propres enfants. Il ne peut se rajeunir qu'en faisant table rase de son propre passé. Un droit concret qui se targue de son existence pour prétendre à une durée illimitée, éternelle ressemble à l'enfant qui lève la main sur sa propre mère. Il insulte à l'idée du droit en l'invoquant, car l'idée du droit est éternellement le devenir ". ${ }^{(9)}$ Le droit mue, s'écoule incessamment. C'est un flot ininterrompu, un bouillonnement de phénomènes. ${ }^{(10)}$ Toutefois, il y a dans le droit une partie fixe à côté de la partie en remous. La partie fixe est d'abord dans la structure du droit. La partie mobile montre l'évolution du droit en accord avec son époque, il est alors un des éléments de la santé de la nation : "C'est le fouet et c'est le sabot de la diligence ". L'idée d'un droit évolutif permet de récuser le mal héréditaire, les traditions, l'habitude, pour faire germer le droit de l'avenir.

La loi de 1898 vient s'inscrire dans les brisures des codes Napoléon en comblant les parties mortes. Sous l'empire du code pénal de 1810, comme l'indiquent les articles $309,310,311$, les parents n'étaient châtiés
(7) Julien BONNE-

CASE, L'Ecole de l'exégèse en droit civil, éd. 1924.

(8) E. PICARD,

Les Constantes du droit, Paris, Flammarion, 1909, p. 143.

(9) Von IHERING,

Le Combat pour le droit, traduit de l'allemand par François Meydieu, employé de l'Etat à l'ambassade de France à Vienne, Paris, Durand et Pedone Lauriel, 1885.

Traduit aussi, sous le titre La Lutte pour le droit, par O. de Meulenaere, conseiller à la Cour d'appel de Gand, Paris, Maresq, 1890.

(10) Gabriel TARDE, Les Transformations $d u$ Droit, Paris, Alcan, 1893. 
(11) J.O., Doc. parl., Chambre des députés, 16 avril 1897, Annexe n 2192, p. 10. sévèrement de leurs mauvais traitements que s'il en était résulté pour l'enfant une incapacité de travail ou une maladie. En l'absence donc de mutilation, d'amputation ou de privation de l'usage d'un membre, de cécité, de perte d'un œil ou d'autres infirmités permanentes, de mort enfin, l'article 309 ne pouvait s'appliquer. L'article 310 augmentait la pénalité édictée par l'article précédent, quand il y avait eu préméditation ou guetapens. Or, font remarquer les promoteurs de la loi, un père dénaturé martyrise son enfant quand l'idée lui en vient, sans rime ni raison; il ne méditera pas à l'avance ce qu'il fera subir à son fils. Il n'y aura donc jamais préméditation au sens strict du mot; de guet-apens encore moins, car il suppose de la résistance. Henri Cochin remarque que « ces articles édictent une peine dérisoire, établissent des distinctions qui ne peuvent guère s'appliquer aux enfants ". ${ }^{(1)}$ Dans l'affaire Machicoine (12 août 1892), le docteur Vibert est contraint de conclure : "Les inculpés n'ont pas tué leur enfant; il est mort de pleurésie purulente et de pneumonie; ces affections ne sauraient être considérées comme la conséquence directe des coups portés à l'enfant, mais ces mauvais traitements sont cause que les maladies ont fait de rapides progrès et ont pu devenir mortelles ». Et Machicoine n'est condamné qu'à six mois de prison, sa femme à deux ans. C'est qu'Henri Cochin dépose en 1897 une proposition de loi modifiant les articles 310 et 311 du code pénal comme il suit : "Lorsqu’il y aura eu préméditation ou guet-apens ou lorsque les violences ou voies de fait auront été commises sur la personne d'un enfant au-dessous de l'âge de quinze ans accomplis, d'une manière habituelle, par des ascendants ou toutes autres personnes auxquelles les enfants se trouvaient confiés, la peine sera, si la mort s'en est suivi, celle des travaux forcés à perpétuité; si les violences ont été suivies de mutilation, amputation ou privation de l'usage d'un membre, cécité, perte d'un oeil ou autre infirmité permanente, la peine sera celle des travaux forcés ".

\section{AU NOM DE LA MORALE}

C'est au nom d'un idéal moral que le législateur républicain fabrique du fait social et définit le statut du pauvre, du criminel, de l'enfant. Pour les entrepreneurs de moralité, ligueurs et membres d'œuvres dont René 
Bérenger (1815-1914) est l'exemple type, la politique n'existe et n'atteint son but que grâce à l'afflux constant de la sève morale. La morale est, au XIXe siècle, la question politique par excellence, la condition préalable à la résolution du problème social sous l'ensemble de ses aspects, et l'on sait le parti qu'en tireront les pères fondateurs de la Troisième République pour laïciser la société civile. ${ }^{(12)}$ L'histoire du malheur de l'enfant prend la forme d'une parole prophétique, religieuse ou laïque, qui entretient avec elle des rapports de passion. Toutefois, dans l'esprit des réformateurs moraux, le rapport qui existe entre la morale et la religion ne peut être ramené à une simple coexistence de fait, ni à une identité ou à une contradiction conceptuelle; c'est un rapport souple et vivant, qui s'impose à ceux qui scrutent l'idée de solidarité. La loi morale propose essentiellement la réalisation de l'harmonie sociale et c'est un objectif tout à fait tangible. Théophile Roussel, René Bérenger, fervents catholiques, républicains, portent sur la scène parlementaire et au Sénat le récit de la souffrance de l'enfant. Dans la violence qui lui est faite, ce qui est souligné, c'est la nature de l'homme, ses passions, ses faiblesses. Le combat moral en faveur de l'enfance se légitime par des causes multiples : conservation de la race, ménagement des forces en vue du service militaire, crainte du paupérisme, enfance coupable. Jacques Bonzan, dans sa thèse portant sur la Législation de l'enfance (1898), décrit le bataillon des natures grossières et avilies "promptes à se laisser mener là où leur est promise une jouissance à leur portée ". Le champ des croisades morales au XIXe siècle est immense : absinthe, pornographie, prostitution, crime, dépopulation, dégénérescence, infanticide, homosexualité, écrits séditieux. Le militantisme moral de la "Belle Epoque" est dominé en France - mais pas uniquement - par un déferlement de panique multiforme, une angoisse dont l'origine n'est pas facilement identifiable, mais dont les manifestations sont spectaculaires. La France, imagine-t-on, est un pays menacé de disparition par la dégénérescence de sa population. Alors que Vachet de Lapouge, avec d'autres eugénistes, propose la stérilisation obligatoire des "êtres béréditairement inférieurs", alors que la République prend conscience de la nécessité de son intervention dans les domaines de l'hygiène et de la santé publique, en
(12) Annie STORA-

LAMARRE (dir.),

Colloque Incontournable morale, Besançon, 9-

10 octobre 1997, à paraître dans Annales littéraires de Franche-Comté. 
(13) Francis RONSIN,

La Grève des ventres; propagande néo malthusienne et baisse de la natalité en France, XIXe-XXe, Paris, Aubier, 1980.

(14) H. DUMERIL De la puissance paternelle et de la protection de l'enfance, Paris, 1881, p. 3.

(15) Raoul de la GRASSERIE, Les Principes sociologiques $d u$ droit civil, Paris, V. Giard et Brière, 1906. quelques années, surgissent par dizaines des associations militantes dont l'objectif est de stopper cette décadence et d'impulser la mobilisation pour la régénération de ce qu'on appelle alors volontiers la "race". ${ }^{(13)}$ C'est au nom de la morale que Paul Robin fonde, en 1896, la Ligue de la régénération humaine et que naît l'Alliance nationale pour l'accroissement de la population française dirigée par Jacques Bertillon. En 1882, le pasteur Fallot crée la Ligue française de la moralité publique. Le sénateur René Bérenger fonde, en 1894, la Société de protestation contre la licence des rues qui réussit à réunir en congrès à Bordeaux, en 1905, 3.000 représentants des différents mouvements de défense de la moralité publique. Mais la morale n'est pas seulement un discours; elle est un programme. C'est en cela que la République fonde la modernité. Il convient de faire de l'ordre avec du désordre, de fonder des règles à un moment où l'anthropologie juridique est à la recherche d'une science de l'écart, du crime et de la maladie. Le remède consiste à élargir les cadres juridiques de manière à y faire entrer l'homme tout entier. Le pauvre, le criminel, l'enfant abandonné, martyrisé, le fou ne sont plus seulement des figures du mal ou du malheur; tous sont sous le regard du droit. Dans ce monde en crise, où les appétits sont surexcités par une civilisation malade, la figure de l'enfant sert à exalter la marche en avant de l'Humanité. Il faut le protéger et l'éduquer. Dans sa thèse de droit datée de 1881, le juriste Duméril cite Guizot, pour qui le XVIIIe siècle a pris fait et cause pour tous les opprimés : les serfs, les hérétiques, les aliénés. Duméril affirme que continuer l'œuvre des pères, c'est se pencher sur une classe intéressante de victimes, les enfants "pauvres petits êtres, adolescents chétifs et souffreteux, vivant au milieu d'une atmosphère malsaine et exposée à la contagion du vice ". ${ }^{(1)}$ L'attention se porte sur les singularités du sujet, indique l'irruption d'une différence et de l'écart. Le juriste catholique Raoul de la Grasserie, membre de la Société générale des prisons, voit le soi comme étant à la racine de la fondation du lien civil. Il distingue dans le soi plusieurs éléments; le droit sur le soimême qui est le droit à la vie. ${ }^{(15)}$ Ce droit est complet, l'autonomie se nomme alors l'inviolabilité de la vie humaine. Aucune personne ne peut acquérir un droit sur la vie, ni par force, ni par convention. Les atteintes 
à la vie doivent être sanctionnées par la société. Les droits de la société contre le soi entament la liberté par l'emprisonnement, l'honneur par les peines infamantes, le domicile par le bannissement et privent l'individu peu à peu de toute autonomie. Le droit d'ingérence sur le soi ouvre à des problèmes moraux. L'homme a-t-il le droit de se tuer? A-t-il le droit de se mutiler ou de se faire souffrir sans utilité pour lui? A-t-il celui de risquer sa vie dans un duel? Peut-il se dégrader moralement et physiquement par l'ivresse, la débauche, tous les vices? A-t-il le droit de se ruiner par le jeu et les prodigalités? Le soi se décompose en plusieurs séries. Il contient des droits irréductibles, la santé, la liberté, l'honneur. Les coups, les lésions, les blessures y portent atteinte, de même les aliments nuisibles ou les violations de l'intégrité sexuelle. Cette histoire du soi ouvre à une pathologie du droit civil mettant en scène le sujet. L'enfant naturel est dans une situation d'indignité vis-à-vis de la société entière; il l'est encore vis-à-vis de la famille et là, même reconnu, il est affecté d'incapacités nombreuses. Il en est de même de certaines catégories d'enfants naturels, des incestueux, des adultérins, des enfants naturels nés avant le mariage et reconnus depuis, ou encore d'enfants victimes d'attentat à la pudeur. Henri Joly, auteur de La France criminelle, président de la Croix Blanche (1900), de la Ligue contre l'athéisme (1895), de la Société Générale des Prisons (1905), de la Société de patronage des jeunes libérés de la petite Roquette (1907), observe que la force fait place à la ruse, à mesure que l'homme avance dans la vie. Il y a prédominance des attentats à la pudeur sur les enfants dans la vieillesse. Le tort le plus fréquent des hommes plus âgés, dit Joly, c'est de prétendre être jeunes. Quand ils s'irritent de ne plus l'être et quand ils veulent à tout prix se comporter comme s'ils l'étaient, ce tort devient aisément un crime : cinquante deux fois sur cent, les viols et attentats à la pudeur sur les enfants sont commis par des hommes de plus de quarante ans. À partir de ces mises en tutelle, de ces atteintes au corps de l'enfant, il faut imposer l'idée de réparation. En 1888, devant le Sénat, Théophile Roussel déclare : «En matière de protection de mineurs contre des parents indignes, l'essentiel est d'organiser la garde de la personne ». ${ }^{(1)}$ Le législateur part du principe que l'enfant, être humain, membre de la société, doit être protégé dans son inté-

(16) Théophile ROUSSEL, Sénat, ler mai 1888. 
(17) Michel FOU-

CAULT, Surveiller et

punir. Naissance de la prison, Paris, Gallimard, 1975. Michel FOU-

CAULT, "La poussière et le nuage", in Michelle PERROT, L'Impossible prison, 1980, p. 29-30. Marcel GAUCHET, L'Inconscient cérébral, Paris, Seuil, 1992. grité physique et morale et que les pouvoirs publics ont le droit et l'obligation d'intervenir pour assurer sa protection.

\section{LE MOUVEMENT D’INDIVIDUALISATION}

La dislocation des valeurs demande l'enfantement d'une morale qui doit inverser le pessimisme pour accéder à la maîtrise d'un monde nouveau où l'enfant est la figure centrale, figure fixée dans le singulier de la norme ou du mythe. Michel Foucault montre qu'en histoire il s'agit de faire surgir une "singularité". Ce n'était pas si évident que les fous soient reconnus comme des malades mentaux; ce n'était pas si évident que la seule chose à faire avec un délinquant, ce fut de l'enfermer; ce n'était pas si évident que les causes de la maladie soient à chercher dans l'examen individuel du corps. Cette analyse faite de la pratique de l'incarcération pénale ou encore de la folie comme " événement " s'applique à l'enfant : ce n'était pas si évident de prendre conscience de sa souffrance et d'en faire un sujet juridique singulier pour permettre l'avènement de la société des individus émancipés. ${ }^{(17)} \mathrm{Ce}$ paradoxe constitue le fait générateur de l'anthropologie démocratique. Il s'agit, pour Marcel Gauchet, de rendre le surgissement de la figure du scindé de lui-même à son inscription dans le devenir. La recomposition du sujet politique atteint, en cette fin de siècle, un degré critique sur tous les plans, économique, politique ou privé, avec la famille, avec la citoyenneté, au travers du salariat, mais également de par le nouvel espace-temps de la ville et du voyage, des communications et des masses. La morale joue un rôle dans cette mue dramatique des identités, dans la transformation du statut de l'enfance, de la distribution des âges, de la prise en compte de sa souffrance.

Les juristes de la fin du XIXe siècle ont participé à la matrice de toutes les psychologies, sociologies, psychiatries, psychanalyses, bref de ce qu'on appelle les sciences de l'homme en élaborant une philosophie juridique de l'individualisation de la peine. Raymond Saleilles (18551912) doit sa renommée de juriste en matière pénale à l'un des livres jugés comme des plus hardis de la fin du XIXe siècle, L'Individualisation 
de la peine (1898). ${ }^{(18)}$ Dans la seconde moitié du XIXe siècle, la sociologie est née, la psychologie fait d'immenses progrès et le droit prend une orientation nouvelle, Ribot est, en France, le principal représentant de la nouvelle psychologie, grâce à ses études sur l'hérédité psychologique, sur les maladies de la mémoire, de la personnalité et de l'attention, qui confirment l'importance attribuée par Taine à l'étude des cas maladifs et des anomalies. Précurseurs, les travaux de l'école anthropologique italienne modifient profondément la notion de responsabilité morale. Lombroso pose, dès 1876, dans L'Uomo delinquente, les principes de l'anthropologie criminelle. Raymond Saleilles, adversaire de Lombroso, estime que le criminel, ce dissemblable et comme dissocié, a les mêmes sensations intellectuelles que le reste de ses semblables; son cerveau fonctionne normalement, il comprend, il raisonne juste; seule sa conscience sent différemment. Il est donc impossible de mettre cette anomalie de conscience sur le compte d'une perturbation physique ou morbide. L'anomalie est interne, constitutive de son individualité propre et de son caractère. En ce sens, la théorie de Saleilles montre que le besoin individualisé est l'axe positif de la puissance intérieure qui distribue de la justice, élargit la face privée dans de nouvelles indépendances. L'individualisation de la peine doit être recherchée par l'application d'une politique appropriée aux circonstances, plutôt que par l'application d'une loi purement abstraite, ignorante des espèces qui lui seraient soumises. Ce principe de l'individualisation se lit dans la loi sur la répression des violences du 19 avril 1898. Le juge peut se mouvoir entre les limites les plus étendues. Un an de prison et seize francs d'amende marquent le premier échelon de la peine qui peut s'élever jusqu'à la mort, en passant successivement par la réclusion et les travaux forcés. Il est désormais possible de proportionner la peine au délit en tenant compte de toutes les circonstances qui l'ont accompagné. La gradation de la pénalité ne repose plus sur les effets du délit mais sur l'intention du délinquant. La réforme avait été vivement sollicitée par le Cinquième congrès international pénitentiaire, tenu à Paris en 1895. De la discussion, il était ressorti que le nouveau critérium aurait l'avantage suivant : il n'était plus besoin que l'enfant mourût; il suffisait que l'in- 
tention homicide de ses bourreaux fût prouvée, quels qu'en eussent été les effets, pour que le châtiment suprême fût encouru. Les alinéas 5 et 6 de l'article premier portent : "Si les blessures, les coups ou la privation d'aliments ou de soins ont été suivis de mutilation, d'amputation ou privation de l'usage d'un membre, de cécité, perte d'un cil ou autres infirmités permanentes, ou s'ils ont occasionné la mort sans intention de la donner, la peine sera celle des travaux forcés à perpétuité ».

\section{LE DROIT DE CITATION OU L'ARTICULATION PUBLIC/PRIVÉ}

La lacune vient de ce que l'œuvre de charité privée ne participe pas complètement à l'œuvre de justice. Or, c'est à l'intérieur des domiciles privés que la férocité se donne cours, protégée par la loi qui rend les foyers domestiques inviolables et sacrés. Qui va donc prendre la défense de l'enfant? Qui va révéler les tortures dont il est l'objet? Un seul voisin est capable d'affronter de pareils dangers; il se nomme Don Quichotte. Le réformateur moral désigne les associations de défense de l'enfance. Le 16 février 1898, la Société générale des prisons émet le vœu que les associations charitables soient investies du droit de citer directement en police correctionnelle les auteurs de violences envers les enfants. L'Union française pour le sauvetage de l'enfance, la Société protectrice de l'enfance, le Patronage de l'enfance et de l'adolescence, la Ligue fraternelle des enfants devront être reconnus d'utilité publique. Au Sénat, les sénateurs Strauss et Théophile Roussel s'en font les défenseurs convaincus. Ils proposent un article additionnel à l'article 6 de la loi ainsi conçu : "Le droit de poursuivre et de porter partie civile peut être concédé par décret spécial, après avis du Tribunal de première instance, aux associations protectrices de l'enfance reconnues d'utilité publique pour ce qui touche les violences et les attentats commis contre les enfants. Ce droit sera exercé pour chaque association par un de ses membres spécialement désignép par le Garde des sceaux et assermenté ». Cette proposition fut repoussée, par crainte "qu'à l'ombre de ces institutions qui existent actuellement, il ne se fonde des sociétés 
d'un autre genre, qui prendront en main cette arme redoutable et pourront ainsi peser dans la vie du citoyen d'un poids excessivement lourd ". ${ }^{(19)}$ L'élargissement des attributions politiques en matière sociale ne veut pas dire multiplication des contrepoids, comme c'est le cas aux Etats-Unis et en Angleterre. Les juristes réunis à la Société générale des prisons précisent qu'il existe en Angleterre et aux Etats-Unis de grandes sociétés à qui l'on a conféré le droit de citer directement devant les juges les parents indignes. ${ }^{(20)}$ Ces sociétés jouissent d'un droit d'avertissement. Les lacunes de la loi vont servir à penser l'articulation entre le domaine national et international dans une démarche comparatiste.

\section{LE RÔLE DU DROIT INTERNATIONAL}

Les progrès du droit comparé font apparaitre les lacunes de la législation française. L'idée de codifier le droit des gens n'est plus conçue comme un rêve, mais comme une preuve de la force nouvelle du droit international et comme un progrès répondant aux besoins du temps. "La terre s'ouvre à l'universalisme des transactions ». Pour Saleilles, la mission créatrice du droit comparé est de fournir aux sociétés un instrument efficace de rapprochement et d'unification générale. L'histoire comparative révélera, dans les données de droit national, les éléments issus d'un développement historique spécial à ce droit. La politique juridique indiquera la diversité des conditions nationales d'application. ${ }^{(21)}$ De ce matériau, il faut alors déduire la synthèse. Les fondateurs de la loi de 1898 ont des carrières internationales. Odilon Barrot (1841-1904) entre dans la diplomatie avec le titre d'attaché d'ambassade dès 1858 . Secrétaire d'ambassade en 1865, il exerce ses fonctions successivement à Bruxelles, Madrid et Washington et devient ensuite attaché à la direction politique du ministère des Affaires étrangères à Paris en 1867. Les juristes cherchent des modèles d'application dans les codes européens. Dans le code pénal des Pays-Bas, l'âge de la victime est considéré comme une circonstance aggravante, lorsque cette victime est âgée de moins de sept ans. La définition des mauvais traitements est également 
(22) J.O., Rapport au Sénat. Doc. parl., 1898, annexe 69, p. 102. très large : c'est le fait de nuire à la santé. Dans la législation britannique, les anglais, "gens pratiques", ont vu une circonstance aggravante dans le fait que la personne coupable avait droit à percevoir une somme d'argent en cas de mort de l'enfant, s'il est constaté qu'il connaissait ce fait. La loi espagnole du 26 juillet 1878 punit d'emprisonnement les parents qui ne donnent pas à leurs enfants les aliments et vêtements nécessaires. En Hollande, la jeune fille est protégée contre la séduction jusqu'à l'âge de 16 ans et la peine du séducteur est même augmentée si elle a moins de douze ans. En Allemagne, c'est la même limite de seize ans. En Angleterre également; et, si la jeune fille est âgée de moins de treize ans, toute relation sexuelle avec elle peut être punie des travaux forcés à perpétuité. La Hongrie, elle aussi, a reconnu un délit dans le fait de se livrer à un commerce sexuel avec une jeune fille âgée de moins de quatorze ans. Est-il possible d'imiter ces législations étrangères? Aussi Georges Berry propose-t-il d'ajouter à l'article 334, qui prévoit des peines de 6 mois à 3 ans de prison en répression des attentats aux mœurs, le paragraphe suivant : "Celui qui dans le but de satisfaire ses passions se sera rendu complice de l'acte de corruption sur un enfant de moins de seize ans sera condamné à la même peine ". ${ }^{(22)}$

Ainsi la loi du 19 avril 1898 montre-t-elle de nouvelles incriminations, dont la plus puissante est sans doute la reconnaissance du mal, plus encore, de "l'effrayant" commis sur le corps de l'enfant. Le délit est certes ancien, mais à la fin du siècle le législateur fait passer à l'état d'infraction divers actes dont il ne s'était pas ému jusque-là. Est-ce dû à la difficulté plus grande d'accepter la souffrance morale et physique? Nietzsche, dans Humain, trop Humain, dit que les Anciens cherchaient à oublier ou bien à infléchir leur sentiment dans le sens de l'agréable : en cela, ils recherchaient l'aide de palliatifs, alors que les hommes de la fin du siècle s'attaquent directement aux causes du mal et préferent en somme agir prophylactiquement. Peut-être, conclut Nietzsche, s'agit-il de poser les fondations sur lesquelles les hommes de l'avenir rebâtiront aussi le temple de la joie? 